\title{
ApoE Deficiency Compromises the Blood Brain Barrier Especially After Injury
}

\author{
Nassia Methia, ${ }^{1,2 *}$ Patrick André, ${ }^{1,2 *}$ Ali Hafezi-Moghadam, ${ }^{1,2}$ Maria Economopoulos, ${ }^{1}$ \\ Kennard L. Thomas, ${ }^{1}$ and Denisa D. Wagner ${ }^{1,2}$ \\ ${ }^{1}$ The Center for Blood Research \\ ${ }^{2}$ Department of Pathology, Harvard Medical School, Boston, MA, USA \\ Contributed by D. Wagner. Accepted November 11, 2001
}

\begin{abstract}
Background: Apolipoprotein E (apoE) mediates lipoprotein uptake by receptors such as the LDL receptor (LDLR). The isoform apoE4 has been linked to Alzheimer's disease and to poor outcomes after brain injury. Astrocytes that induce blood brain barrier (BBB) properties in endothelium also produce apoE. We decided to investigate the role of apoE in BBB function and in the restoration of BBB after brain injury.

Materials and Methods: Wild-type (WT) mice and mice deficient in apoE or LDLR were fed normal chow or diets rich in fat and cholesterol. The BBB leakage was determined through injection of Evans blue dye and measurement of the amount of dye extravasated in the brains 3 hours later. Brain injury was induced by applying dry ice directly onto the excised parietal region of the brain. The mice were given 7 days to recover. In some experiments, peroxidase was infused to observe the site of leakage by histology.

Results: We found $70 \%$ more spontaneous leakage of injected Evans blue dye in the brains of apoE $-/-$ mice than
\end{abstract}

\section{Introduction}

Mice lacking apoE or low density lipoprotein receptor (LDLR) provide excellent genetic backgrounds to study development of atherosclerotic lesions (1). While evaluating the role of various endothelial adhesion molecules in atherosclerosis, we noted that mutations in the adhesion genes when combined with apoE-deficiency, but not with LDLR-/- deficiency, frequently lead to premature death. This occurred often in the first day of life, as if caused by birth trauma, with hemorrhage sometimes showing in the head. We started to question whether apoE was important for brain vasculature, particularly since apoE is produced by astrocytes (2) whose endfeet are in close proximity to the brain endothelium. Astrocytes induce in the endothelium blood brain barrier (BBB) properties $(3,4)$. Thus we decided to examine the role of apoE in vascular permeability

\footnotetext{
Address correspondence and reprint requests to: Denisa D. Wagner, The Center for Blood Research, 800 Huntington Avenue, Boston, MA, 02115. Phone: (617) 278-3344; fax: (617) 278-3368; e-mail: wagner@cbr.med.harvard.edu. ${ }^{*}$ N. Methia and P. André contributed equally to this work.
}

in wild type. This increase in permeability appeared selective for the brain. The leaky BBB in apoE-/- mice may provide an explanation for the neurological deficits seen in these animals. In an established model of BBB leakage induced by trauma (cold injury), the apoE- - mice showed even more compromised BBB function, compared with WT mice, suggesting that apoE is important for BBB recovery. No deficit in BBB was observed in injured LDLR-/- mice, even on Western Diet. In contrast, higher plasma cholesterol levels in apoE- $/-$ mice further increased BBB leakage after injury. We extracted $5 x$ more Evans blue from these brains than from WT. In the injury model, injection of peroxidase resulted in prominent retention of this protein in the cortex of apoE-/- but not in WT.

Conclusions: Our results show that the combination of loss of apoE function with high plasma cholesterol and especially brain injury results in dramatic BBB defects in the cortex and may explain in part the importance of apoE in Alzheimer's disease and in successful recovery from brain injury. and in the maintenance of BBB, particularly after brain injury.

ApoE clearly plays an important role in the brain. ApoE-/- mice are cognitively impaired $(5,6)$ and show signs of neurodegeneration in the central nervous system (7). Humans have three common isoforms of apoE (apoE2-4) produced by alleles at a single gene locus. Genetic epidemiologic studies have shown that the presence of apoE4 is a risk factor for Alzheimer's disease as well as for poor outcome following brain injury $(8,9)$. The latter linkage is directly relevant to our proposed studies evaluating apoE function in brain vasculature repair. Similarly, closed head injury in apoE $-/-$ mice resulted in more severe motor and cognitive deficits than seen in wild-type mice (10) and increased neuronal damage in apoE- $/-$ mice occurred after transient global cerebral ischemia (11). Following brain injury, the synthesis of apoE is increased and it is secreted by the astrocytes into the extracellular space (12) but its exact role(s) in the brain is not known. In this study we present in vivo evidence that apoE helps to maintain BBB and that its function is of particular importance after brain injury, when BBB needs to be rapidly restored. 


\section{Materials and Methods}

Mice

Wild type and apoE knockout mice (13) were 6-8 weeks old, on C57BL/6J background and obtained from The Jackson Laboratory, Bar Harbor, Maine. Experimental protocols were approved by the Animal Care and Use Committee of the Center for Blood Research.

Vascular Permeability/BBB Leakage of Evans Blue Dye

Evans blue dye was injected intraperitoneally (50 $\mu \mathrm{g}$ per g body weight) and 3 hours later mice were sacrificed and brain or skin from the flank and organs collected and weighed. The specimens were extracted in $3 \mathrm{ml}$ pure formamide (Sigma, St. Louis, MO) for 72 hours (brain) or 90 hours (other tissues) and optical density of the extracted dye measured at $620 \mathrm{~nm}$.

\section{Cold Injury (14)}

Cold injury was performed by applying dry ice directly onto the excised parietal region of the brain for 5 sec. The excised skin was then clipped and Evans blue dye injected 7 days later as described above. The dye was extracted from the injured brains in the same way as from uninjured brains.

\section{Infusion of ${ }^{111}$ Indium-labeled Red Blood Cells}

Red blood cells were incubated with $500 \mu \mathrm{Ci}{ }^{111} \mathrm{In}$ (NEN Life Science Products, Boston, MA) for $30 \mathrm{~min}$ at $37^{\circ} \mathrm{C}$. Washed red blood cells $\left(1 \times 10^{7}\right.$ in $200 \mu \mathrm{l}$ saline) were injected into the tail vein. One hour later blood was collected by retroorbital puncture and brain and left kidney harvested and cpm determined in a $\gamma$-counter (Model 1185, Searle Analytic, Des Plaines, IL).

\section{Mouse Diets}

Normal mouse chow contains 5\% fat (wt/wt) and 0.019\% cholesterol (Prolab 3000, PMI Nutrition International, St. Louis, MO). The "enriched" diet TD 01021 containing $10.5 \%$ fat and $0.1 \%$ cholesterol and the "Western Diet" TD 88137 containing $21.2 \%$ fat and $0.2 \%$ cholesterol were obtained from Harlan Teklad, Madison, WI. Mice were on the various diets for two weeks before and during the 7 days of the cold injury experiment. Cholesterol levels were determined after two weeks on the diet by using a "cholesterol 20" kit (Sigma).

\section{Horse Radish Peroxidase Deposition in the Cortex}

Mice were injected via tail vein 2 times with $10 \mathrm{mg}$ horseradish peroxidase (Sigma) in $300 \mu \mathrm{l}$ of PBS at time 0 and at 3 hours. Three hours after the second injection the brains were rinsed by cannulating both carotid arteries with heparinized PE10 tubings connected to heparinized $30 \mathrm{ml}$ syringes containing PBS. A syringe pump was set at a rate of $1 \mathrm{ml} / \mathrm{min}$ to drive the syringes and perfuse PBS through the carotid arteries while simultaneously causing blood to exit the jugular veins during the procedure. The rinsing was continued until there was no visible reddish hue to the fluid exiting the jugular veins. Thereafter, $30 \mathrm{ml}$ of $2 \%$ paraformaldehyde (Sigma) was perfused through the brain at the same rate. Subsequently, the cranium was carefully opened and the brains harvested and fixed with $4 \%$ paraformaldehyde at $4^{\circ} \mathrm{C}$ over night. The cortex was cut into $1-2 \mathrm{~mm}$ thick slabs, embedded in 14\% Agar and sectioned $150 \mu \mathrm{m}$ thick slices). The slices were incubated for $20 \mathrm{~min}$ at room temperature in 0.05M Tris- $\mathrm{HCl}$ buffer, $\mathrm{pH} 7.6$, with $5 \mathrm{mg}$ of 3-3'diaminobenzidine (Sigma) and $0.01 \%$ hydrogen peroxide per $10 \mathrm{ml}$ of buffer to reveal peroxidase activity, and photographed through a light microscope. The quality of washing was controlled by the absence of dark red blood cells containing endogenous peroxidase activity.

\section{Results}

Effect of ApoE-deficiency on Systemic Blood Vessel Permeability and Blood Brain Barrier Permeability

To evaluate blood vessel leakage, apoE-/- (13) and wild-type (WT) mice on C57BL/6J background, 6-8 weeks old, were injected intraperitoneally with Evans blue dye. Evans blue dye binds to albumin and they permeate out of normal vessels into surrounding tissues but not where $\mathrm{BBB}$ is in place (15). Three hours after the injection, systemic change in skin color was observed and mice were sacrificed and skin and several organs or brains were harvested and the dye extracted. Surprisingly, we consistently observed about $70 \%$ more dye extracted from the apoE $-/-$ brains than from WT brains (Fig. 1), while the amounts of dye extracted from the skin and other organs/per gram of tissue were not different (Fig. 2). Thus it appears that the absence of apoE selectively increases permeability of vessels in the brain.

To rule out the possibility that the blood vessels were larger or more numerous in the apoE-/- brains than in WT brains, leading to more dye accumulation, we injected via tail vein ${ }^{111}$ In-labeled red blood cells (they do not permeate out of vessels) and the mice were sacrificed 1 hour later. The radioactivity in the brain was determined and normalized to radioactivity of a blood sample obtained from the same animal (Table 1). The results show that the brain blood volumes of WT and apoE-/- mice were the same and therefore the Evans blue had to permeate and accumulate outside the vessels in the ApoE-/- brains.

\section{Blood Brain Barrier Recovery after Cold Injury of Cortex} of Mice Fed Normal Chow Diet

To evaluate the capacity of apoE-/- mice to repair BBB function we used a cold injury model $(14,16)$ with mice 8 weeks old. Cold injury is an established model for traumatic brain injury followed by vasogenic brain edema. It induces a rapid BBB breakdown, 


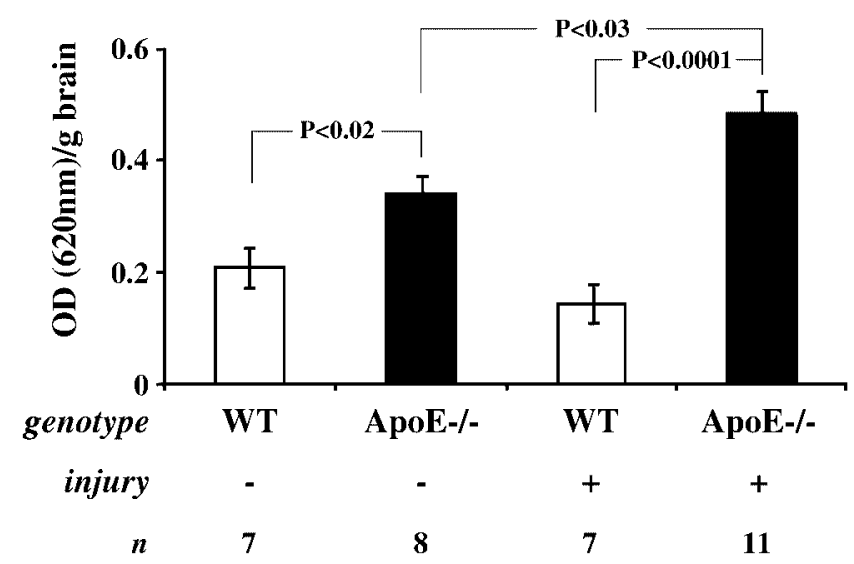

Fig. 1. Increased blood brain barrier permeability in apoE $-/-$ mice. 8 weeks old WT and apoE- $-1-$ mice were injected with Evans blue dye. Three hours later the mice were sacrificed and the dye extracted from the brains (injury -). Alternatively, the mice were subjected to cold injury of the cortex seven days prior to dye injection (injury + ). ApoE $-I-$ mice had significantly increased BBB permeability to Evans blue dye bound to albumin and this difference from WT was accentuated after brain injury. Optical density of brains obtained from animals without Evans blue injection was negligible.

reflected by an immediate increase in Evans blue leakage. This leakage is short-lived and returns to nearly control levels by 24 hrs in WT mice (16). We applied a small piece of dry ice directly onto the skull in the left parietal region and the mice were allowed to live for an additional 7 days. Three hours before sacrifice, the mice were injected with Evans blue. After the cold injury, the brains of WT mice accumulated the same amount of dye as the uninjured WT brains (Fig. 1), indicating that the damage produced by the injury was repaired fully during the 7 day healing period and BBB was reestablished. In contrast, the injured apoE-l-brains contained more than three times as much dye as the injured WT brains and also more dye than the uninjured apoE $-/-$ brains (Fig. 1). This shows that the brain

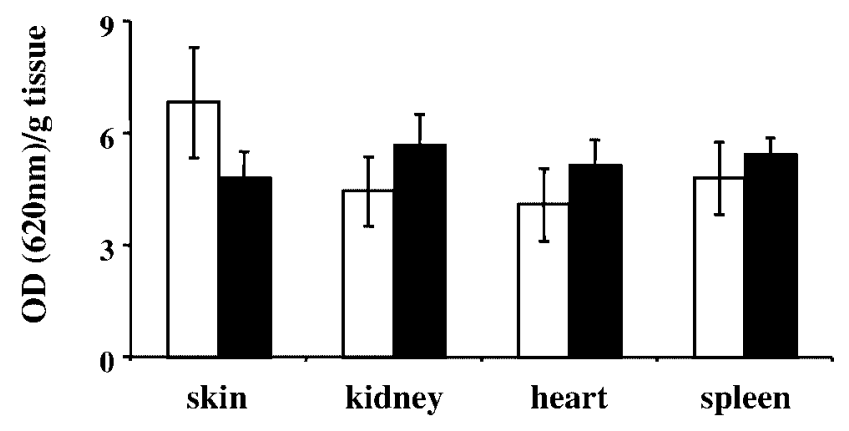

Fig. 2. Permeability of peripheral vessels in apoE-/- mice. Wild-type mice (open bars) and apoE $-I-$ mice (black bars) were injected with Evans blue dye and sacrificed 3 hours later. Optical density of the dye extracted from skin and indicated organs was measured. No differences in vascular permeability between the two genotypes were found $(n=6-8 ; P=0.28-0.62)$.
Table 1. Comparison of blood volume in brains of WT and apoE $-/-$ mice

\begin{tabular}{|c|c|c|}
\hline & \multicolumn{2}{|c|}{ Genotype } \\
\hline & WT & ApoE-/- \\
\hline \multicolumn{3}{|l|}{ Cpm/g brain } \\
\hline Cpm/40 $\mu$ l blood & $1575.7 \pm 305.4$ & $1485.4 \pm 369.9$ \\
\hline $\mathrm{n}$ & 6 & 6 \\
\hline$P$ value & \multicolumn{2}{|c|}{0.85} \\
\hline
\end{tabular}

injury of the apoE mice further aggravated the observed leakage through the BBB.

\section{Effect of Cholesterol Level in Plasma on the Extent of Blood Brain Barrier Leakage After Cold Injury}

There is a significant physiological difference between WT and apoE-/-mice that could affect blood vessel function. The apoE mice present about two-three fold higher cholesterol level than WT mice, leading to spontaneous atherosclerotic lesion formation $(13,17)$. Although the young mice used in this study do not develop significant atherosclerotic lesions, their high cholesterol level could possibly be the cause of the observed BBB defect. To evaluate whether high cholesterol induces BBB leakage per se, we used different diets causing elevation of plasma cholesterol in LDLR-/- or apoE-/- mice (Fig. 3). If high cholesterol antagonizes BBB formation rather than the absence of apoE, then the BBB leakage should be similar among mice with similar cholesterol values. This is not what we observed.

LDLR - /- mice on normal chow have slightly higher plasma cholesterol $(230 \mathrm{mg} / \mathrm{dl})$ than WT (18) and without injury their brains contained the same amount of dye as WT brains (WT $0.209 \pm 0.035$; LDLR $-/-0.171 \pm 0.029 ; \mathrm{n}=7-8 ; P=0.4)$. After cold injury, BBB permeability had a tendency to increase slightly in the LDLR-/- mice as compared with WT injured brains (WT $0.142 \pm 0.034 ;$ LDLR $-/-0.282 \pm$ $0.039 ; \mathrm{n}=7-13, P=0.07)$ but this did not reach statistical significance. LDLR $-/-$ mice put on two different high cholesterol/fat diets for two weeks prior to injury responded by increasing significantly cholesterol in their plasma but their BBB permeability was not modified from injured LDLR $-/-$ mice on normal chow (Fig. 3). This indicates that high cholesterol alone does not cause BBB breakage. In contrast, the cholesterol level produced by Western Diet in apoE-/- and LDLR-/- mice were comparable (approx. $1350 \mathrm{mg} / \mathrm{dl}, P=0.7$ ), but the injured apoE $-/$ - brains contained significantly more Evans 

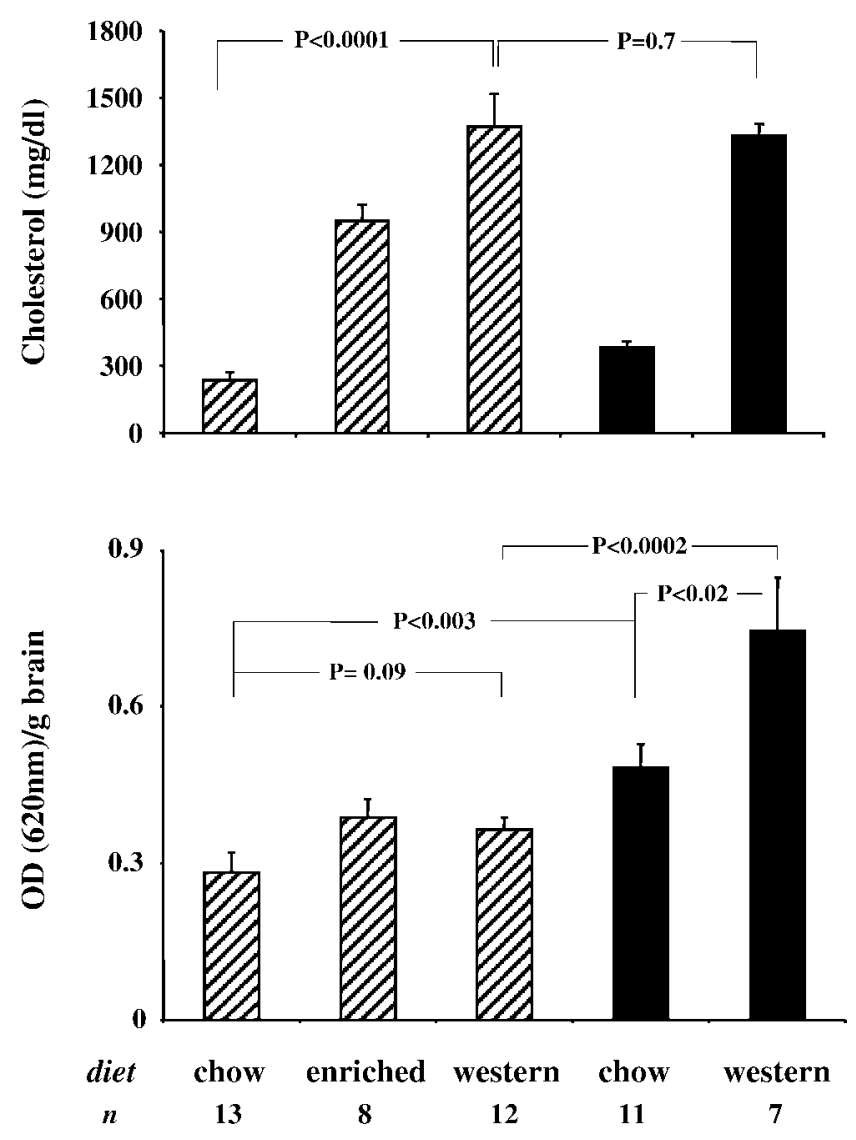

Fig. 3. Effect of high plasma cholesterol on BBB permeability following brain injury. LDLR $-/-$ mice (striped bars) and apoE $-/-$ mice (black bars) were fed mouse chow (chow), enriched diet (enriched) or Western Diet (western) for 2 weeks prior to cold injury to the cortex and during the 7 day healing period. (Top) The diets produced different total plasma cholesterol levels in these mice. (Bottom) Mice on these diets were sacrificed 3 hours after Evans blue injection and the dye was extracted from their brains. In the LDLR-/- mice, the increasing cholesterol levels did not affect the amount of dye in the brain while the injury-induced leakage of BBB in the apoE-lmice increased in the presence of high plasma cholesterol.

blue than the LDLR brains $(P=0.0002)$. Interestingly, in the apoE-/- mice the Western Diet further aggravated the BBB leakage after injury indicating that, in the absence of apoE, high cholesterol can be a contributing factor to increased BBB leakage after injury to the cortex.

\section{Visualization of Blood Brain Barrier Leakage with Horseradish Peroxidase}

Uninjured and 7 days post injury mice were injected via tail vein with peroxidase (19). Six hours later the brains were perfused through carotid arteries with buffer to remove from the vessels the remaining peroxidase and red blood cells that have strong endogenous peroxidase activity. The brains were processed and reacted with 3-3'diaminobenzidine and the gray matter of cerebral cortex examined by light microscopy. In the uninjured apoE-/- cortex

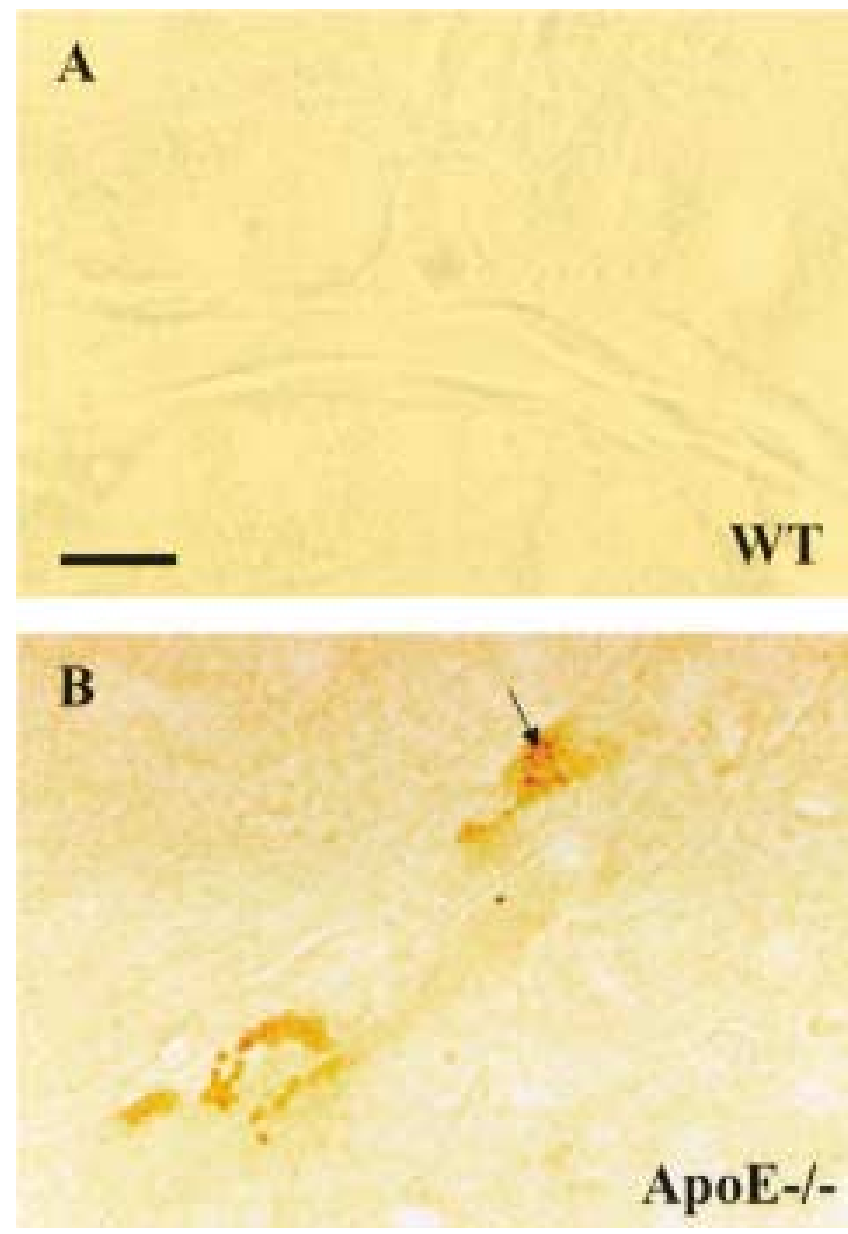

Fig. 4. Permeation of peroxidase from blood vessels of the cortex after injury. Seven days after cold injury to the cortex, WT (A) and apoE-I- (B) mice were injected i.v. with peroxidase and 6 hours later the intravascular peroxidase was flushed out and brains were processed and stained for peroxidase activity. While almost no positive staining was seen in WT sections, the apoE-l- showed strong deposits of peroxidase periodically distributed along the blood vessels (arrow). A weaker staining could be observed already one hour after a peroxidase infusion in the knockout brains (not shown). Bar $=30 \mu \mathrm{m}$.

sporadic small dark deposits were observed associated with the vascular structures. These were almost never seen in WT cortex (not shown). The deposits became much more prominent after injury in the apoE-/-brains, periodically surrounding the blood vessels (Fig. 4B). The peroxidase staining remained very rare in WT injured brains (Fig. 4A). A blinded observer could genotype the sections by the intensity of peroxidase staining. This indicates that only in the apoE-/- mice brains does the peroxidase penetrate and accumulate in places whence it could not be washed out by perfusion.

\section{Discussion}

The results presented here show that: 1) apoEdeficiency causes the formation of a leaky BBB as detected by excessive albumin extravasation in the 
brains of young adult mice; 2) the defect is selective to brain vessels, as there is no difference in permeability in other vessels we examined; 3 ) injury to the cortex significantly aggravates the subsequent leakage of the $\mathrm{BBB}$, indicating that apoE is crucial for effective $B B B$ recovery and; 4) high cholesterol, when combined with apoE-deficiency but not separately, further aggregates BBB leakage after injury. Thus the combination of apoE-deficiency with injury and a high fat/cholesterol diet, produced a five-fold higher albumin leakage than seen in injured WT brains.

During the preparation of this manuscript two studies were published that observed sporadic spontaneous leakage of $\mathrm{BBB}$ in apoE $-/-$ mice. The first study, by Fullerton et al. (20), detected the presence of extravascular IgG in the spinal cord and in cerebellum of approximately half of the young apoE $-/-$ mice examined. Infused Evans blue was also detected by fluorescence microscopy in the hippocampus of the apoE $-/-$ mice. The second study by Mulder et al. (21), also observed IgG immunoreactivity in the brains of old apoE $-/-$ mice, but not in 3 month old animals. The lower prevalence of BBB permeability in uninjured young adult brains reported in both of these publications than in ours likely reflects a lesser sensitivity of the immunostaining technique in comparison with measuring the optical density of the extracted dye. Seven $/ 8$ apoE $-/$ - brains gave optical density of the dye higher than the mean of WT brains. We have also observed leakage of peroxidase in the cortex of uninjured young mice (not shown)where BBB normally is very tight (19). By not detecting leakage in young but seeing it easily in old apoE $-/-$ mice, the study by Mulder et al. shows that BBB permeability in the apoE $-/-$ mice increases with age.

Our original contribution to these studies is showing the importance of apoE in the restoration of BBB after cortical injury. Since our analyses were quantitative, we could compare the relative intensity of BBB leakage under the different scenarios. The amount of dye in uninjured apoE brains was higher only by a fraction than that found in WT brains, while after injury the difference was several fold (Fig. 1). Since the injury was to the cortex, it can be assumed that it was there where the injuryinduced leakage was located. Indeed this was confirmed by the peroxidase extravasation in that region, examined 7 days post injury (Fig. 4).

Thus the results of the three independent studies together show that apoE-loss of function alone or combined with brain injury, high cholesterol and/or old age significantly increases BBB permeability. Interestingly, these are the major risk factors established for Alzheimer's disease (9). It is therefore likely that leaky BBB is a contributing factor in this devastating disorder. ApoE clearly affects the extent of amyloid- $\beta$ deposition in the brain (hallmark of Alzheimer's disease) (22). The breaks in BBB seen in apoE $-/-$ mice might induce more amyloid- $\beta$ to leak from blood to the brain (23), leading to more amyloid accumulation over a lifetime; or interfere with clearance of the peptide from the brain by active transport across the BBB (24). It will be interesting to examine $\mathrm{BBB}$ leakage in apoE$-/-$ mice producing the human isoforms of the protein (25), we would not be surprised if mice expressing the apoE4 protein approximated the null phenotype.

The efficient restoration of blood brain barrier is also important for patients with traumatic head injury or cerebral ischemia. BBB leakage of proteinrich fluid in these conditions results in brain edema (26). In its extreme form edema leads to compression of the brain stem leading to respiratory arrest.

How does apoE contribute to the development or maintenance of BBB? We consider it unlikely that it is just by regulating lipid metabolism. LDLR-/mice also have significant defects in lipid metabolism and are able to repair BBB function as well or almost as well as WT mice-even in the presence of the Western Diet (Fig. 3). ApoE could have a signaling function upon the brain microvasculture inducing tight junction formation or inhibiting transcellular vesicular traffic seen in other endothelial beds (4). Such signaling would be unlikely to go exclusively through LDLR since the LDLR-/- do not have leaky BBB. Perhaps the LDLR-related protein (LRP) could be the signaling partner as it is also expressed by brain endothelium and polymorphisms of this receptor were recently implicated in Alzheimer's disease $(27,28)$.

The function of apoE in the restoration of the BBB after brain injury may be more complex than its function in the maintenance of the barrier. Superoxide radicals play an important role in pathogenesis of cold injury as the resulting edema is reduced in transgenic mice overexpressing CuZn-superoxide dismutase $(16,29)$. Interestingly, apoE may function as an antioxidant (22). ApoE was shown to protect a neuronal cell line from hydrogen peroxide-induced cytotoxicity (30). Thus, after a brain injury, apoE may both limit the extent of the injury and help in restoration of the BBB. An open question is the origin of the apoE important for the BBB restoration. Astrocytes secrete basic fibroblast growth factor (31) and yet unidentified small molecule(s) (32), that promote $\mathrm{BBB}$ formation and they could provide the necessary apoE as well. Since endothelium is in contact with blood, the apoE could also originate from the liver and act from the apical side.

Clearly separating the central nervous system from blood is of major importance. The absence of apoE affects permeability of proteins to the brain, it remains to be seen whether the other function of $\mathrm{BBB}$, the ionic barrier, is supported by apoE as well. The potential roles of various endothelial adhesion and junctional proteins in the BBB formation and repair should now be addressed in vivo as their role may be highlighted in combination with the apoEdeficient background. 


\section{Acknowledgments}

We thank Dr. Morris Karnovsky for stimulating discussions and advice, Dr. Richard O. Hynes for critical reading of the manuscript, and Lesley Cowan for help with the preparation of the manuscript. This work was supported by National Heart, Lung and Blood Institute, National Institutes of Health grant R01 HL 53756 (to D.D.W.).

\section{References}

1. Breslow JL. (1996) Mouse models of atherosclerosis. Science 272: 685-688.

2. Boyles JK, Pitas RE, Wilson E, et al. (1985) Apolipoprotein E associated with astrocytic glia of the central nervous system and with nonmyelinating glia of the peripheral nervous system. J. Clin. Invest. 76: 1501-1513.

3. Janzer RC, Raff MC. (1987) Astrocytes induce blood-brain barrier properties in endothelial cells. Nature 325: 253-257.

4. Rubin LL, Staddon JM. (1999) The cell biology of the bloodbrain barrier. Annu. Rev. Neurosci. 22: 11-28.

5. Gordon I, Grauer E, Genis I, et al. (1995) Memory deficits and cholinergic impairments in apolipoprotein E-deficient mice. Neurosci. Lett. 199: 1-4.

6. Oitzl MS, Mulder M, Lucassen PJ, et al. (1997) Severe learning deficits in apolipoprotein E-knockout mice in a water maze task. Brain Res. 752: 189-196.

7. Masliah E, Samuel W, Veinbergs I, et al. (1997) Neurodegeneration and cognitive impairment in apoE-deficient mice is ameliorated by infusion of recombinant apoE. Brain Res. 751: 307-314.

8. Strittmatter WJ, Roses AD. (1996) Apolipoprotein E and Alzheimer's disease. Annu. Rev. Neurosci. 19: 53-77.

9. Roses AD. (1997) Apolipoprotein E, a gene with complex biological interactions in the aging brain. Neurobiol. Dis. 4: 170-185.

10. Chen Y, Lomnitski L, Michaelson DM, Shohami E. (1997) Motor and cognitive deficits in apolipoprotein E-deficient mice after closed head injury. Neuroscience 80: 1255-1262.

11. Horsburgh K, Kelly S, McCulloch J, et al. (1999) Increased neuronal damage in apolipoprotein E-deficient mice following global ischaemia. Neuroreport. 10: 837-841.

12. Poirier J. (1994) Apolipoprotein E in animal models of CNS injury and in Alzheimer's disease. Trends Neurosci. 17: 525-530.

13. Zhang SH, Reddick RL, Piedrahita JA, Maeda N. (1992) Spontaneous hypercholesterolemia and arterial lesions in mice lacking apolipoprotein E. Science 258: 468-471.

14. Kakinuma Y, Hama H, Sugiyama F, et al. (1998) Impaired blood-brain barrier function in angiotensinogen-deficient mice. Nat. Med. 4: 1078-1080.

15. Wolman M, Klatzo I, Chui E, et al. (1981) Evaluation of the dye-protein tracers in pathophysiology of the blood-brain barrier. Acta Neuropathol. 54: 55-61.

16. Murakami K, Kondo T, Yang G, Chen SF, Morita-Fujimura Y, Chan PH. (1999) Cold injury in mice: a model to study mechanisms of brain edema and neuronal apoptosis. Prog. Neurobiol. 57: 289-299.

17. Plump AS, Smith JD, Hayek T, et al. (1992) Severe hypercholesterolemia and atherosclerosis in apolipoprotein Edeficient mice created by homologous recombination in ES cells. Cell 71: 343-353.

18. Ishibashi S, Brown MS, Goldstein JL, Gerard RD, Hammer RE, Herz J. (1993) Hypercholesterolemia in low density lipoprotein receptor knockout mice and its reversal by adenovirus-mediated gene delivery. J. Clin. Invest. 92: 883-893.

19. Reese TS, Karnovsky MJ. (1967) Fine structural localization of a blood-brain barrier to exogenous peroxidase. J. Cell Biol. 34: 207-2 17 .

20. Fullerton SM, Shirman GA, Strittmatter WJ, Matthew WD. (2001) Impairment of the blood-nerve and blood-brain barriers in apolipoprotein e knockout mice. Exp. Neurol. 169: 13-22.

21. Mulder M, Blokland A, van den Berg DJ, et al. (2001) Apolipoprotein E protects against neuropathology induced by a high-fat diet and maintains the integrity of the bloodbrain barrier during aging. Lab. Invest. 81: 953-960.

22. Fagan AM, Holtzman DM. (2000) Astrocyte lipoproteins, effects of apoE on neuronal function, and role of apoE in amyloid-beta deposition in vivo. Microsc. Res.Tech. 50: 297-304.

23. Maness LM, Banks WA, Podlisny MB, Selkoe DJ, Kastin AJ. (1994) Passage of human amyloid beta-protein 1-40 across the murine blood-brain barrier. Life Sci. 55: 1643-1650.

24. Shibata M, Yamada S, Kumar SR, et al. (2000) Clearance of Alzheimer's amyloid-ss(1-40) peptide from brain by LDL receptor-related protein-1 at the blood-brain barrier. J. Clin. Invest. 106: 1489-1499.

25. Raber J, Wong D, Buttini M, et al. (1998) Isoform-specific effects of human apolipoprotein $\mathrm{E}$ on brain function revealed in ApoE knockout mice: increased susceptibility of females. Proc. Natl. Acad. Sci. U.S.A. 95: 10914-10919.

26. Kempski O. (2001) Cerebral edema. Semin. Nephrol. 21: 303-307.

27. Herz J, Gotthardt M, Willnow TE. (2000) Cellular signalling by lipoprotein receptors. Curr. Opin. Lipidol. 11: 161-166.

28. Van Uden E, Kang DE, Koo EH, Masliah E. (2000) LDL receptor-related protein (LRP) in Alzheimer's disease: towards a unified theory of pathogenesis. Microsc. Res. Tech. 50: 268-272.

29. Chan PH, Yang GY, Chen SF, Carlson E, Epstein CJ. (1991) Cold-induced brain edema and infarction are reduced in transgenic mice overexpressing $\mathrm{CuZn}$-superoxide dismutase. Ann. Neurol. 29: 482-486.

30. Miyata M, Smith JD. (1996) Apolipoprotein E allele-specific antioxidant activity and effects on cytotoxicity by oxidative insults and beta-amyloid peptides. Nat. Genet. 14: 55-61.

31. Sobue K, Yamamoto N, Yoneda K, et al. (1999) Induction of blood-brain barrier properties in immortalized bovine brain endothelial cells by astrocytic factors. Neurosci. Res. 35: 155-164.

32. Dehouck B, Dehouck MP, Fruchart JC, Cecchelli R. (1994) Upregulation of the low density lipoprotein receptor at the blood-brain barrier: intercommunications between brain capillary endothelial cells and astrocytes. J. Cell Biol. 126: 465-473. 